\title{
The Model Coupling Toolkit
}

\author{
J. Walter Larson ${ }^{1}$, Robert L. Jacob ${ }^{1}$, Ian Foster ${ }^{1}$, and Jing Guo ${ }^{2}$ \\ 1 Argonne National Laboratory, Mathematics and Computer Science Division \\ 9700 S. Cass Ave., Argonne, IL 60439, USA, \\ larson@mcs.anl.gov \\ ${ }^{2}$ Data Assimilation Office, NASA Goddard Space Flight Center \\ Greenbelt, MD 20771, USA
}

\begin{abstract}
The advent of coupled earth system models has raised an important question in parallel computing: What is the most effective method for coupling many parallel models to form one high-performance coupled modeling system? We present our solution to this problem-The Model Coupling Toolkit (MCT). We describe how our effort to construct the Next-Generation Coupler for NCAR Community Climate System Model motivated us to create the Toolkit. We describe in detail the conceptual design of the MCT, and explain its usage in constructing parallel coupled models. We present some preliminary performance results for the Toolkit's parallel data transfer facilities. Finally, we outline an agenda for future development of the MCT.
\end{abstract}

\section{Introduction}

In recent years, climate modeling has evolved from the use of atmospheric general circulation models (GCMs) to coupled earth system models. These coupled models comprise an atmospheric GCM, an ocean GCM, a dynamic-thermodynamic sea ice model, a land-surface model, and a flux coupler that coordinates data transfer between the other component models, and governs the overall execution of the coupled model (Figure11). Coupled models present a considerable increase in terms of computational and software complexity over their atmospheric model counterparts.

The flux coupler typically serves the following functions: overall command and control of the coupled model, including syncronization, error/exception handling, and intitialization and shutdown of the system; communication of data between component models; time averaging and accumulation of data from one component for use in subsequent transmission of data to other components; computation of interfacial fluxes for component given state data from other components; interpolation of flux and state data between the various component model grids; merging of flux and state data from multiple components for delivery to yet another component. 
The computational demands of each of the component models are sufficient to require message-passing parallelism (and in some cases hybrid parallelism) in each component model to achieve high performance on microprocessor-based distributed memory computers. This creates a formidable challenge in coupling these models together. This challenge manifests itself in a potentially high degree of computational and software complexity in the flux coupler. The coupler must be aware of all the component models, the grids on which they present and require data, and their repsective data decompositions. The coupler must be able to handle all of this information, and serve the information required by the component models in a timely fashion, lest it cause the coupled system to hang.

Various coupled model and coupler architectures have been created in the attempt to meet the aforementioned challenges. A general diagram for coupled model architecture is shown in Figure2, which shows the main parts of a coupled model with four component models as a "wheel": the syncrhonization and command/control apparatus (the "rim" of the wheel); the coupler computatonal core (the "hub" and "spokes" of the wheel); the component models (e.g., atmosphere, ocean, sea-ice, and land); the component model-coupler interfaces.

There are five main architectural approaches for coupled models:

1. A single-executable "event loop" coupled model, in which a single pool of processers us used for the coupled model, with each the component models and the coupler running in turn. Execution of the model under this architecture can be viewed as a sweep second hand revolving around the wheel diagram in Figure 2 An example of this architecture is the Parallel Climate Model (PCM).

2. A single-executable asynchronous coupled model, with each of the component models and coupler runnin simultaneously and exchanging data as needed.

3. A multiple-executable asynchronous coupled model, with each of the component models and coupler runnin simultaneously and exchanging data as needed. An example of this architecture is the current version of the NCAR CCSM.

4. A single-executable asynchrouns model in which the functions of the flux coupler are distributed among the various component models. In terms of Figure 2, the flux coupler (the "hub" and the "spokes" of the wheel) disappear. An example of this coupling strategy is the Fast Ocean-Atmosphere Model (FOAM).

5. A multiple-executable asynchrouns model in which the functions of the flux coupler are distributed among the various component models.

We took as our primary coupler design requirement the ability to support each of the five aforementioned coupled model architectures. 


\section{A Typical Coupled Model}

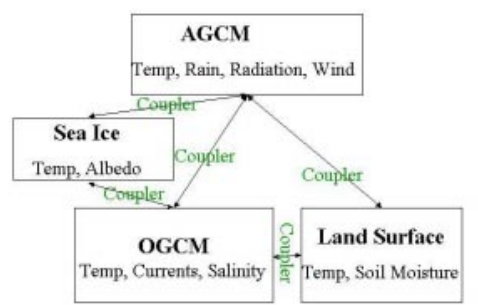

Fig. 1. Schematic of a coupled earth system model, showing the component models and examples of the flux and state data they exchange.

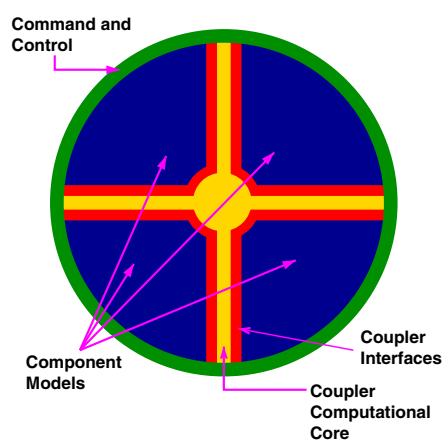

Fig. 2. Schematic of a coupled modeling system, with component models.

\section{The Community Climate System Model Next-Generation Coupler}

The problem that motivated the creation of the model coupling toolkit was the Accelerated Climate Prediction Initiative (ACPI) Avant Garde project, whose goal is the creation of a modular, performance- portable Community Climate System Model (CCSM). One major task in this project is the design and implementation of a modular, extensible, high-performance "Next-Generation Coupler" (NGC) for the CCSM.

A full statement of the requirements for the NGC are given at the URL

http://www.cgd.ucar.edu/csm/models/cpl-ng/\#requirements

Two types of requirements were identified-scientific requirements and computational functionality requirements. The scientific requirements outline the coupler's role in the coupled modeling system, and a list of the core functions the coupler must provide. The computational functionality requirements outline the programming language(s) to which the coupler must provide interfaces, and portability and performance issues. 


\section{Next Generation Coupler Design Layers}

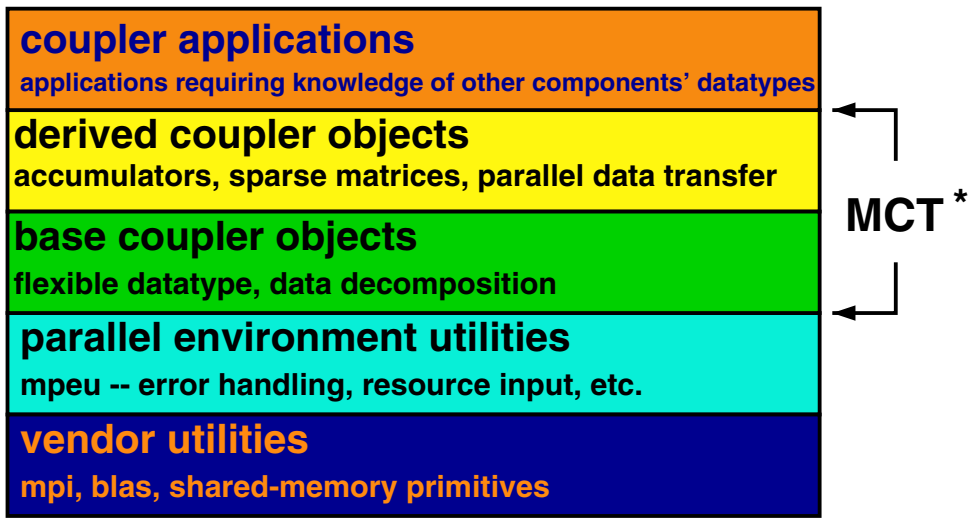

* Model Coupling Toolkit

Fig. 3. Layered design for the CCSM Next-Generation Coupler.

Analysis of the two groups of requirements yielded a layered design. The Layers (Figure 3), ranked lowest-level to highest-level are: Vendor Utilities, which include standard libraries, vendor implementations of the Message-Passing Interface (MPI) library, and shared-memory primitives (e.g., SHMEM); Parallel Environment Utilities, which include the Data Assimilation Office's Message Passing Environment Utilities (mpeu) library, and LBL's Message-Passing Handshaking (MPH) utilities; Basic Coupler Classes and Methods, which include low-level MCT objects such as the internal data representation and data decomposition descriptors; Derived Coupler Classes and Methods, which include the MCT datatypes and routines to support: interpolation implemented as sparse matrix-vector multiplication; time averaging; computation of fluxes from state variables; Coupler Applications, which are the coupler computational core and component model-coupler interfaces built using MCT components, and facilities for converting component model datatypes and domain decomposition descriptors into MCT components.

\section{The Model Coupling Toolkit}

The model coupling toolkit is a set of software components that ease the programming of parallel coupled modeling systems. The main services provided by the toolkit are: data decomposition descriptors; a flexible, extensible, indexible field storage datatype; support for time averaging and accumulation; data field interpolation implemented as sparse matrix-vector multiplication; intercomponent communications and parallel data transfer. 


\subsection{Description and Underlying Assumptions}

The MCT is highly modular, and implemented in Fortran 90. All the toolkit functions feature explicit INTENT declarations in their interfaces and extensive argument and error checking. Toolkit modules and routines have prologues that can be processeed using the tool ProTeX to create LaTeX documentation. Further information regarding the toolkit, including complete documentation is available at the MCT web site:

http://www.mcs.anl.gov/ larson/mct

The MCT relies on the following underlying assumptions: each component has its own MPI communicator; each component has a unique integer ID; each component is on a distinct set of processors; interpolation implemented as sparse matrix-vector multiplication; components can exchange only real and integer data as groups of vectors. The MCT user must supply a consistent numbering schemes for grid points for each component model grid, and the interpolation matrix elements. Once the user has satisfied these assumptions and requirements, the MCT allows the user to link any number of component models, using any grid, any domain decomposition, and any number of processors-per component model.

\section{$3.2 \quad$ Toolkit Components}

The low-level components in the MCT are the internal data field representation and data decomposition descriptors. The high-level components in the toolkit are: a component model registry; time-averaging and accumulation registers; sparse matrix storage; the grid geometry description; the communications scheduler. The high and low-level components are presented in Table 3.2

Fields are represented in the MCT in its own internal data structure called an attribute vector, and is implemented in the AttrVect component. The AttrVect is used extensively in the Toolkit's interpolation, time-averaging, and parallel data transfer functions. The AttrVect component is defined as a Fortran 90 derived type:

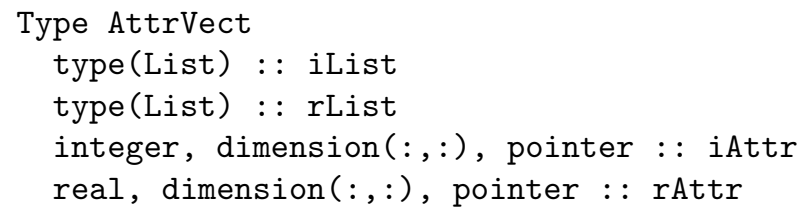

The List components iList and rList list the integer and real attributes (fields) of the AttrVect respectively. A List is a string, with substrings delimited by colons. Suppose we wish to store the real fields for surface zonal and meridonal winds and temperature in an AttrVect component. We first define 
string tags for each field: us for surface zonal wind; vs for surface meridional wind; ts for surface temperature. For this example, the rList component would be rList $=$ 'us:vs:ts'.

These fields can be accessed by using an AttrVect inquiry, and supplying the string tag to reference the desired field.

The AttrVect is a fundamental data type in the toolkit, and is used for other purposes than field storage. Other uses include: time averaging and accumulation registers in the Accumulator component; sparse matrix element storage in the SparseMatrix component; grid point coordinate and area/volume weight storage in the GeneralGrid component.

The MCT has two basic types of domain decomposition descriptors: the GlobalMap, which describes a simple, one-dimensional data decomposition, and the GlobalSegMap, which describes a segmented data decomposition capable of describing multidimensional decompositions of multidimensional grids and unstructured grids. The GlobalMap type is a special, simple case of the more general GlobalSegMap. Users of the MCT must translate their domain decompositions into either the GlobalMap or GlobalSegMap form.

A simple example of how the decomposition of a two dimensional grid is stored in a GlobalSegMap is shown in Figure 4.

The MCT provides numerous facilities for manipulating and exchanging the GlobalMap and GlobalSegMap domain descriptor components, including: intercomponent exchanges of maps; global-to-local index translation; local-to-global index translation.

A full description of the higher-level components of the MCT are beyond the scope of this paper, and we have summarized the components and their methods (excluding the create and destroy methods) are summarized in Table tab:mct.

The one high-level portion of the MCT we will describe here is the parallel data transfer. Parallel data transfer is accomplished by creating a communications scheduler called a Router from the domain descriptors of the source and

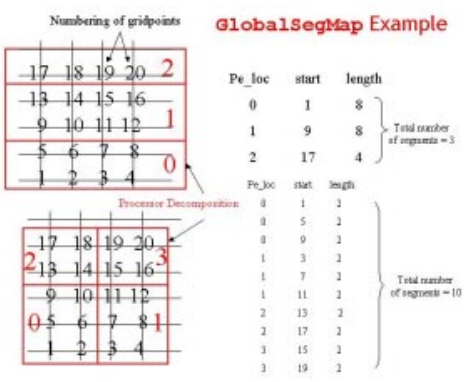

Fig. 4. Illustration of the GlobalSegMap domain decomposition descriptor component. 
target component models. An example of how a Router is created is shown in Figure 5 Once the appropriate Router is created, the parallel transfer is effected by calling the routines MCT_Send and MCT_Recv() on the source and target component models, respectively. The only arguments to these transfer routines are: a Router to coordinate the parallel send (receive); an AttrVect to store the input (ouptut) data.

Table 1. Components of the Model Coupling Toolkit

\begin{tabular}{|c|c|c|}
\hline Service & Component & Methods \\
\hline Data Storage & AttrVect & $\begin{array}{c}\text { Indexing } \\
\text { Sorting } \\
\text { Gather, Scatter, Broadcast }\end{array}$ \\
\hline Domain Decomposition & $\begin{array}{c}\text { GlobalMap } \\
\text { GlobalSegMap }\end{array}$ & $\begin{array}{l}\text { Indexing } \\
\text { Exchange }\end{array}$ \\
\hline Time Average/Accumulation & Accumulator & $\begin{array}{c}\text { Accumulate } \\
\text { methods from AttrVect }\end{array}$ \\
\hline Interpolation & SparseMatrix & $\begin{array}{c}\text { Multiplication } \\
\text { methods from AttrVect }\end{array}$ \\
\hline Grid Description & GeneralGrid & $\begin{array}{l}\text { area/volume integrals } \\
\text { methods from AttrVect }\end{array}$ \\
\hline Component Registry & MCTWorld & $\begin{array}{c}\text { component identificaiton } \\
\text { process address translation } \\
\text { (i.e., from local communicator } \\
\text { to MPI_COMM_WORLD }\end{array}$ \\
\hline Communications Scheduling & Router & $\begin{array}{c}\text { Parallel transfer routines } \\
\text { MCT_Send() and MCT_Recv() }\end{array}$ \\
\hline
\end{tabular}

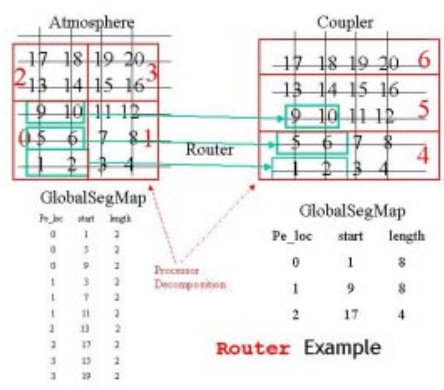

Fig. 5. Illustration of the Router communications scheduler component.

\section{Usage}

Programming of flux couplers and component model-coupler interfaces is accomplished by directly invoking components of the toolkit. A complete example of 
how the MCT is used to create a coupled model is beyond the scope of this article. Instead, we shall focus on the MCT unit tester, which implements the simple example of an atmosphere coupled to and ocean via a flux coupler (Figure 6).

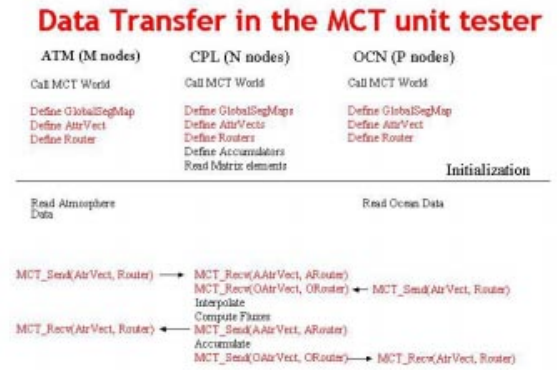

Fig. 6. A simple Atmosphere-Ocean Coupled Model built using the MCT.

\section{Performance}

Performance characterization of the toolkit has just begun, but some preliminary results concerning the most crucial component-the parallel data transfer routines MCT_Send and MCT_Recv-are available. Performance of the parallel transfer is highly sensitive to a number of variables, including the number of MPI processes in the sending and receiving component models, the complexity of the source and target as measured by the numbers of segments in their respective GlobalsegMap descriptors, and the complexity of the interrelationship and overlaps between the source and target domain decompositions. We present prformance results for the transfer of sixteen T42 (128 longitudes by 64 latitudes) atmospheric grid fields between the atmosphere and flux coupler. We present results for two examples that are meant to capture the extremes of the governing performance factors cited above: (1) a simple example in which the number of MPI processes on the atmosphere and coupler are identical, as are their domain decompositions of the atmosphere grid.; and (2) a complicated example in which the atmosphere has many more MPI processes than the coupler and the atmosphere and coupler domain decompositions are not related in any simple fashion.

Case (1) has atmosphere and coupler decompositions as shown in the left and center panels of Figure 7. The performance of MCT_Send and MCT_Recv, as measured on an IBM SP3 $(375 \mathrm{MHz})$, is shown in the right panel of Figure 7 The performance for this simple case is as expected: transfer time decreases as the message size decreases and the number of processors assigned to each model is increased. 

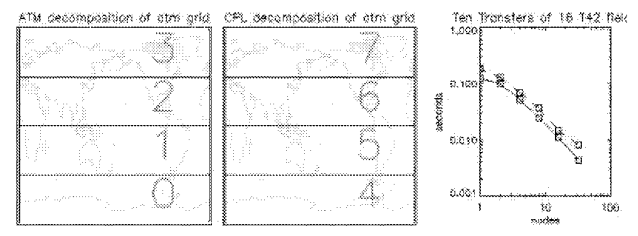

Fig. 7. Atmosphere and Coupler component models with identical domain decompositions.

The domain decompositions for case (2) are shown in the left and center panels of Figure 8. The Router between these two decompositions was automatically determined by MCT. Timing data are shown in right panel of Figure 8 . The number of coupler nodes was varied for each of three cases: with the atmosphere on 8 (black), 16 (red), and 32 (blue) nodes. The poor scaling may be an unavoidable result of doing a parallel data transfer between two very dissimilar decompositions. Still, the overall transfer time is very small compared with the time the full model will spend computing 10 timesteps; moreover, the user/developer is relieved of the burden of determining the complex data transfer pattern.
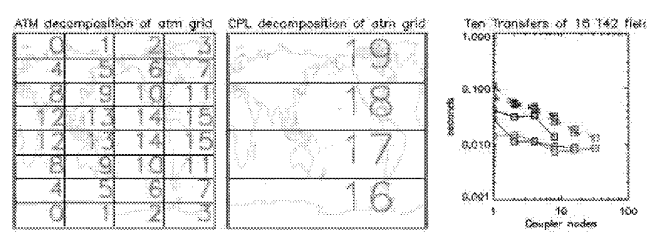

Fig. 8. Atmosphere and Coupler component models with differing numbers of processes and domain decompositions.

Future versions of the toolkit will offer explicit support for dynamic load balancing of component models, assuming the number of MPI processes per component model is held fixed. Accomodating this feature will require the study and optimization of a number of toolkit component methods including: the initialization method for the Router component; initialization methods for the GlobalMap and GlobalSegMap components; domain decomposition descriptor exchange methods. 


\section{Conclusions and Future Work}

A modular Model Coupling Toolkit has been described, and its usage and performance have been discussed. The future development agenda of the MCT includes numerous enhancements: support for on-line interpolation matrix element generation; performance optimization and inclusion of OpenMP to implement hybrid parallelism; upwards abstraction of data types to greater simplify the construction of flux couplers and component model-coupler interfaces; support for higher-dimensional data storage classes; support for higher-dimensional data decomposition descriptor classes; extension to support dynamically load balanced component models (but with fixed process pool sizes); and extension to support dynamically load balanced component models (with dynamically varying process pool sizes).

Acknowledgements: The authors wish to thank many people for the informative and inspiring discussions that helped guide this work: Tom Bettge, Tony Craig, Cecelia Deluca, Brian Kaufman, and Mariana Vertenstein of the National Center for Atmospheric Research; John Michalakes and John Taylor of the Mathematics and Computer Science Division of Argonne National Laboratory. This work is part of the Accelerated Climate Prediction Avant Garde project, and is supported by the Office of Biological and Environmental Research of the U.S. Department of Energy's Office of Science.

\section{References}

[Jones, 1999] Jones, P. W. (1999). First- and Second-order Conservative Remapping Schemes for Grids in Spherical Coordinates. Monthly Weather Reveiw, 127:22042210 . 